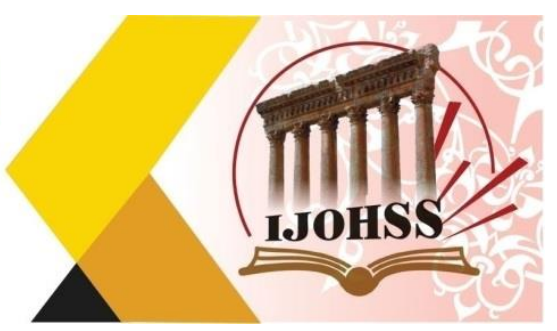

\title{
The Use of Neural Networks to Predict the Number of Gifted Students
}

\author{
Taneem Kamal Aldeen Muhamd Murad \\ Najran University - Kingdom of Saudi Arbia \\ Email: Tasneemmurad1@Hotmail.Com
}

\author{
Nahla Kamal Aldeen Muhamd Murad \\ Najran University - Saudi Arabia \\ nahla.morad@gmail.com
}

\author{
Mustafa Ahmed Salih Elfaki \\ Sudan Academy for Aviation Sciences \\ and Technology (Sudafast) - Sudan \\ 2222elfaki@gmail.com
}

\begin{abstract}
In this study, Neural Network shave been used to classify the views into their groups in the presence of some variables that do not follow natural distribution. This is to identify the most important variables that influence admission at the gifted schools. The criterion of the wrongly rating ratio of viewing has been used as a criterion of the results' accuracy. The study problem has been the method of distinguishing \& classifying gifted students into accepted \& not accepted by the National Board of Schools of Gifted Students whose numbers are increasing in their application to be admitted into those kind of schools, which is creating pressure on the National Board of Schools of Gifted Students. Added to this is the multiplicity of statistical methods to process qualitative data \& the conditions of using each one of them. the study's goal is to identify the most important variables influencing the admission to gifted schools in addition to identifying whether the Neural Networks' method is suitable for processing such data. The study has used the descriptive \& analytical inductive methods by analyzing the study data before formulating the results. The study used the program of Statistical Package of Social Science(SPSS version 20) to process the data. One of the most important results of the study was that the said method was moral \& provided the importance \& effect of the independent variables involved in the analysis. The performance of the Neural Networks was excellent with a good classification of $93 \%$. The most important thing influencing admission was Wexler's Test. Making use of Neural Networks in classifying data was one of the most important recommendations of the Study. The study suggests the introduction of Genetic Algorithm to the Neural Networks.
\end{abstract}

Keywords: Neural Networks, Wechsler test for measuring the intelligence, Classification, Gifted. 


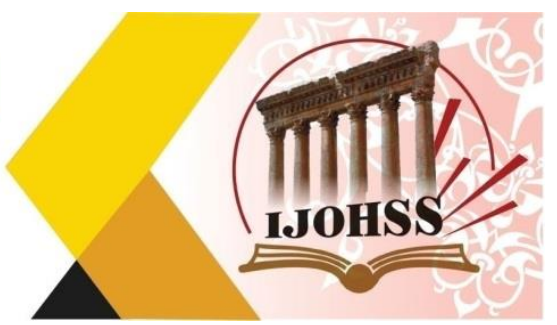

\section{Introduction:}

Artificial Neural Networks is considered as one of the great developments used in the field of the computer. Statistical people have begun to use them with the purpose of prediction. Neural Networks have been used for the purposes of discriminating \&rating in many researches at the intentional level. In 2008, researcher Tahir Raisan Dekhiel used both methods of the Linear Discriminatory Function \& Neural Networks $\&$ got the result that the method of Neural Networks provided better function than the Linear Discriminatory Function ${ }^{1}$. Another research was carried out by Mutter \& Elyas in (2010) "Time Series Analysis and Modeling of Tigris' Monthly Water Flow Running into Mosel City" whereas prediction was carried out using the method of Box-Jinkies \& of Neural Networks ${ }^{2}$.

\section{Study Problem:}

It isin how to discriminate\& classify students admitted to the gifted schools in Sudan under the presence of three stages in the admission exam of gifted schools, making the scientific classification of admission at gifted schoolsin Sudan easier.

\section{Goals of the Study:}

The study aims at identifying the most important variables influencing the admission at gifted schools, identifyingthe method of Neural Networks in the rating of variables, study data variables \& the processing of such data.

\section{Methodology of the Study:}

It is with the aim of achieving the required goals of the study, which has endorsed the analytical inductive method through the method of the Artificial Neural Networks \& the descriptive method of the pattern of the Artificial Neural Networks.

\section{The Community \& Sample of the Study:}

The community is represented in all the students applying for the gifted schools boards in the State of Khartoum, totaling 205 male \& female students.

\section{Used Statistical Tools:}

The wrong rating ratio shall be depended on for the pattern of Neural Networks by applying the SPSS version 20 using models of Neural Networks from the list of Analyze using Multilayer Perception network.

\footnotetext{
${ }^{1}$ Tahir Raisan Dekhiel (2008), the Use of Neural Networks for Discrimination Purposes, a published research paper, Economics \& Management Sciences Magazine, Volume (14), Edition (52) P. 1-7.

${ }^{2}$ Meter, Dhafir, Elyas \& Intisar Ibrahim (2010), "Time Series Analysis and Modeling of Tigris' Monthly Water Flow Running into Mosel City", a comparative study, Iraqi Magazine of Statistical Sciences (18) P. 2-32.
} 


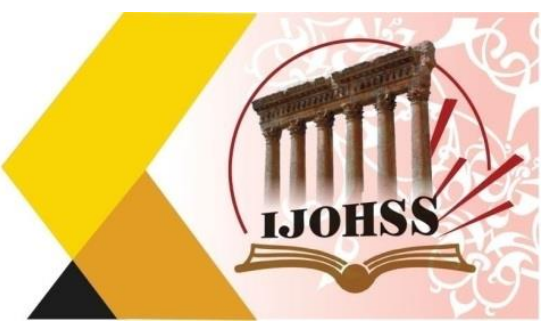

\section{Definition of Artificial Neural Networks (ANN):}

The ANN is a pattern that resembles the natural biological network \& uses a number of basic ways used in natural neurologic systems with the assistance of modeling programs \& the method of parallel processing, i.e. the ANN processes information using the method of imitating the human mind ${ }^{3}$.

\section{The Basic Components of the ANN:}

The ANN is made up of a group of processing units called neural cells or neurons that are identical to the biological neurons of the mind. These units are connected to each other in the form of links called the Neural Network. The links work on the relaying of signs from one neuron to another $\&$ each neuron has an accompanying weight number. Each neuron receives a number of input through its intersections \& produces one output sign only. The output signs move through the way of the outside link of the neuron. In its turn, the output link splits to a number of branches that relay the same sign. The output branching links end at the links coming to other neurons in the Network ${ }^{4}$.

\section{Applying the Method of the ANN: Applied Analysis}

\section{5-5 Description of the Neural Networks Model:}

For the applications of rating by applying the Neural Networks, the Step Function will be used. It is also called the "Threshold Function" because it is suitable for rating seeing that it gives only two results for the resultant ( 0 or 1$)$ as in the following formula:

$f(x)=\left\{\begin{array}{l}0 \text { if } x<0 \\ 1 \text { if } x>0\end{array}\right.$

Whereas the formulaof the function or model becomes as follows:

$f(x)=\frac{1}{1+e^{-s}}$

Whereas the $\mathrm{S}$ represents the scaled total of the input with the (Bios) added to it, which is symbolized with $\theta$, i.e. the scaled total is given by the following formula: $S=\sum_{i=1}^{n} w_{i} x_{i}+\theta$

Whereas $w_{i}$ represents the weights (relative importance of weights) $\& x_{i}$ represents independent variables. Since there are 11 independent variables, the total weighted becomes:

$S=w_{1} x_{1}+\cdots \ldots \ldots .+w_{11} x_{11}+\theta$

Asforthe model, it takes the following form:

\footnotetext{
${ }^{3}$ El-Otaibi, Fozie (2003): "The Use of Time Series \& Neural Networks in the Prediction of Records", a master's thesis in Application Statistics, University of Suez, Port Sayeed.

4 Abdel-Hemied Mohamed El-Ubbasie, 2003, an Introduction to the Artificial Neural Networks \& their Applications in Social Sciences using the SPSS, the Statistical Studies \& Research Institute, Cairo, P. 1-8
} 


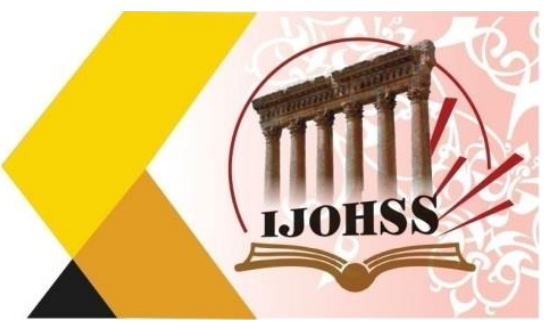

$S=\frac{1}{1+e^{-\left(w_{1} x_{1}+\cdots+w_{11} x_{11}+\theta\right.}}$

\section{Variables of the Study:}

The study includes 11 input variables (independent), which is the place signified by the symbol $\left(\mathrm{x}_{1}\right)$ \&which is Khartoum $=1$, Bahrie $=2$, Omdurman $=3$. Gender is signified by the symbol $\left(\mathrm{x}_{2}\right)$ male $=1$, female $=2$. Age is $\mathrm{V}$, with the symbol $\left(\mathrm{x}_{3}\right)$, seven years, $=1$, eight years $=2$, nine years $=3$. The student's marks in Arabic language have the symbol $\left(\mathrm{x}_{4}\right)$. The marks are divided to successful $=1$, or not successful $=2$. The student's marks in mathematics have the symbol $\left(\mathrm{x}_{5}\right)$. The marks are divided to successful $=1$, or not successful $=2$.

The student's skill in Arabic language has the symbol $\left(\mathrm{x}_{6}\right)$. The marks are divided to successful $=1$, or not successful $=2$. The student's marks in the subject of expression has the symbol $\left(\mathrm{x}_{7}\right)$. The marks are divided to successful $=1$, or not successful $=2$. Verbal capabilities have the symbol $\left(\mathrm{x}_{8}\right)$. The marks are divided to successful $=1$, or not successful $=2$. Mathematical capabilities have the $\operatorname{symbol}\left(\mathrm{x}_{9}\right)$. The marks are divided to successful $=1$, or not successful $=2$. Wexler Test has the symbol $\left(\mathrm{x}_{10}\right)$. The marks are divided to genius $=1$, gifted $=2$, distinguished $=3, \&$ the characters test has the symbol $\left(\mathrm{x}_{11}\right)$. The marks are divided to successful $=1$, or not successful $=2$. As for the dependent variable, which is the acceptance or non-acceptance of the student in the gifted schools, it has the symbol (y). Marks here are divided to accepted $=1$, not accepted $=2$. 


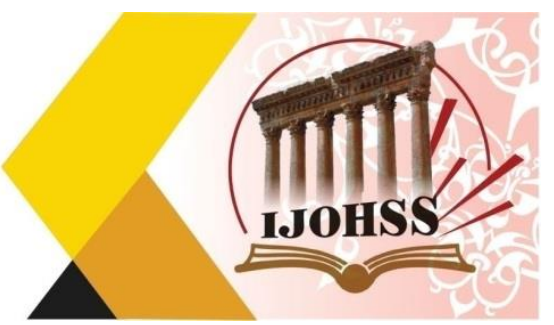

Data Analysis Using the Neural Model:

Network Information

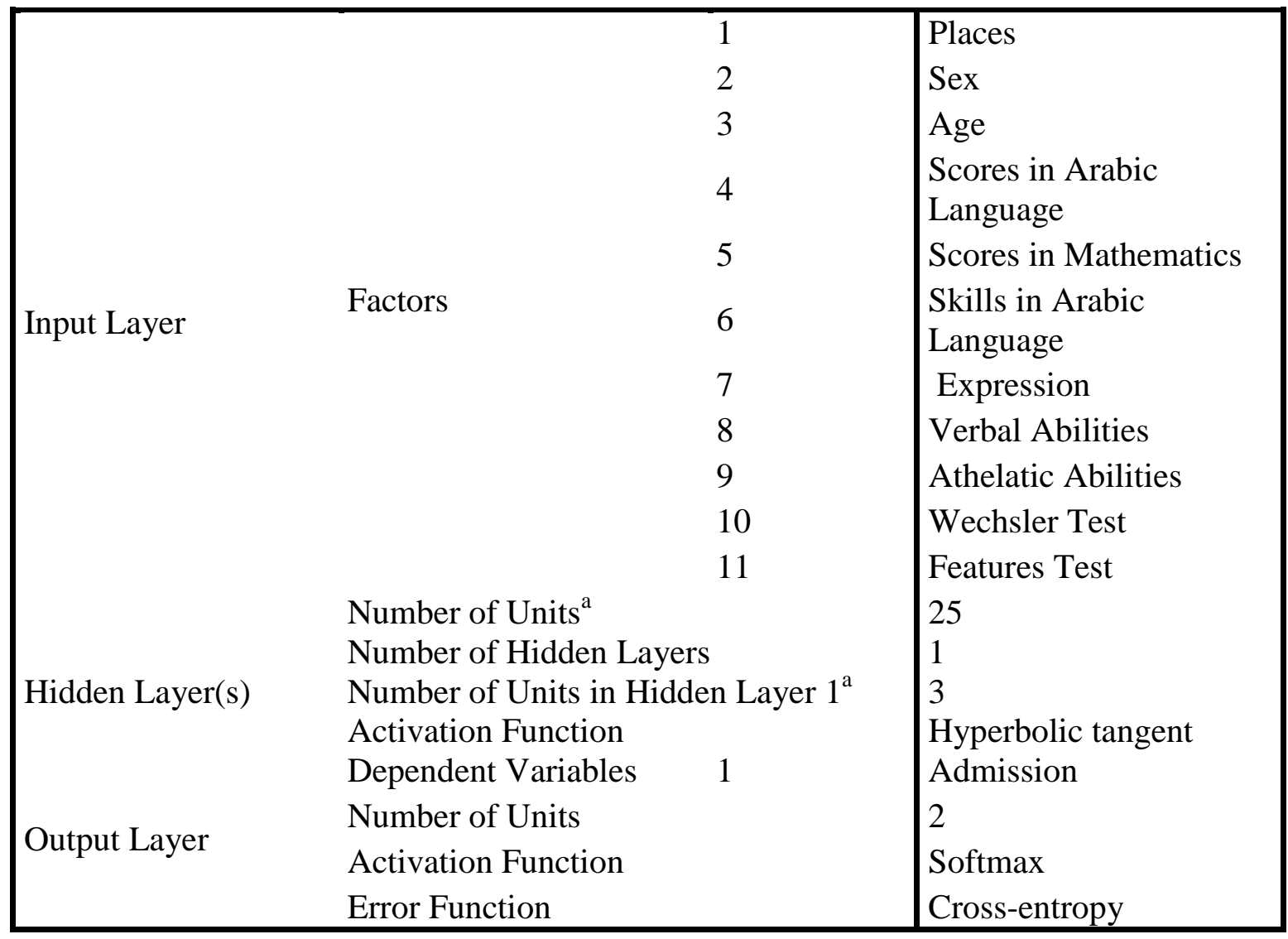

a. Excluding the bias unit

Table (1) usedNeural Networks information

From the above table, we can see that in the part related to the layer of input, there is a variable for each of them both at many levels or factors. There is no covariate, as it is obvious by the input layer $25, \&$ number of units. As for the part related to the hidden layer's information, we find that there is one hidden layer. As for the number of units in the hidden layer, they are twenty five units $\&$ the activation function used in the hidden layer it is the Hyperbolic Tangent Function. There is also one dependent variable, which is Admission. As for the last part of the Table related to the information of the output layer, the estimation has been carried out using the Adjusted Normalized\& the activation function used in this layer is the Softmax Function, which is sometimes known as Sigmoid Function or the Logistic Function. 
المجلة الدولية اللعلوم الأسانية والإمتاعية International Journal on Humanities and Social Sciences website:www.ijohss.com Email:editor@ijohss.com العدد(22) يوليو 2021 ISSN: 2415 - 4822

Volume (22) July 2021

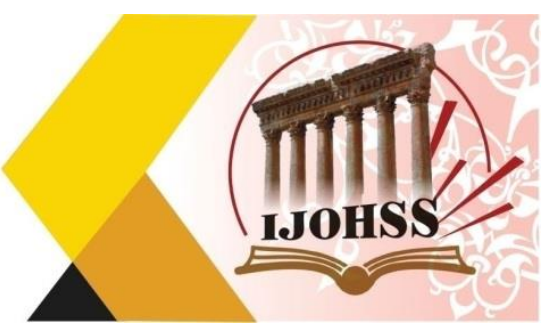

Data shape (1):

Structure of the used Artificial Neural Networks

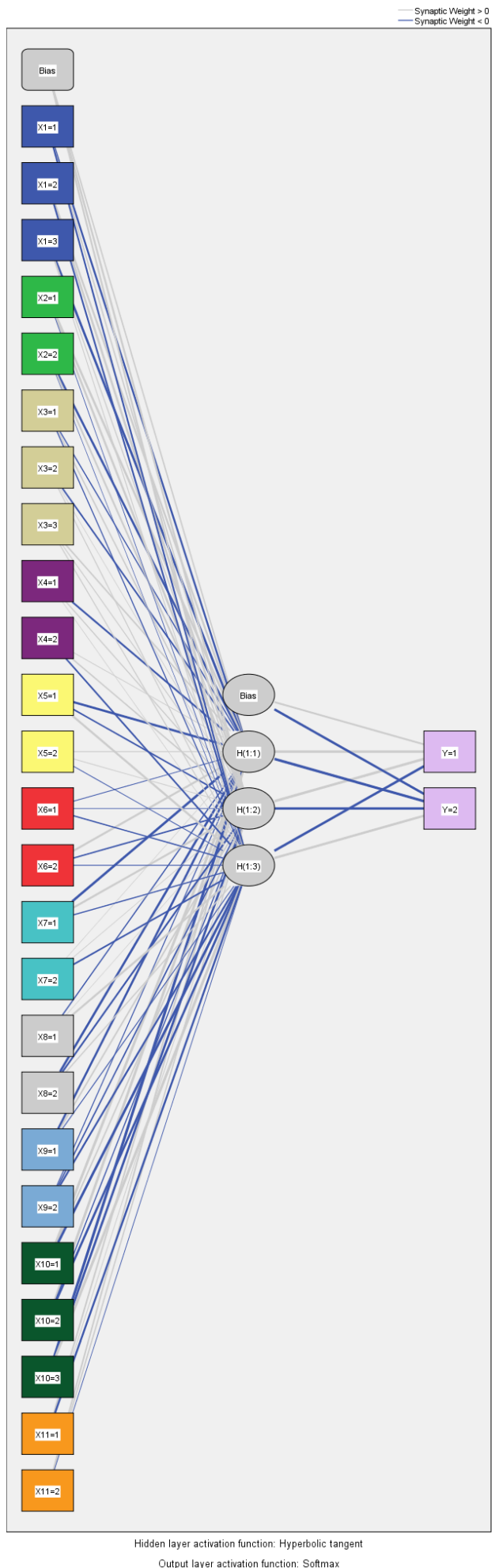




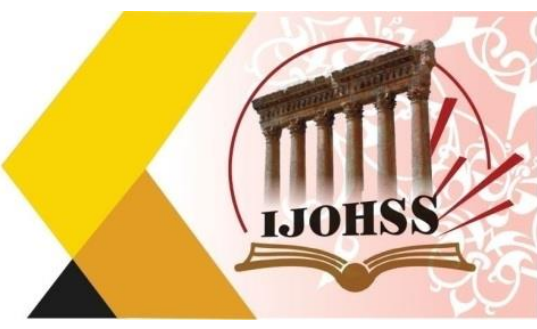

The above graph shows the architecture of the applied Neural Networks that is composed of eleven layers inserted from the left\& is made up of twenty-five units in addition to the bias unit. The second layer is the hidden layer in the center \& is made up of five units. The last layer on the right side is the output layer (resultant) \& they are the accepted at the gifted schools Y.

\section{Model Summary}

\begin{tabular}{|ll|l|}
\hline \multirow{5}{*}{ Training } & Cross Entropy Error & 34.004 \\
& Percent Incorrect & $9.0 \%$ \\
& Predictions & 1 consecutive step(s) with no \\
& Stopping Rule Used & decrease in error \\
& Training Time & $0: 00: 00.25$ \\
& Cross Entropy Error & 10.053 \\
Testing & Percent Incorrect & $6.7 \%$ \\
& Predictions & \\
\hline
\end{tabular}

Dependent Variable: Admission

a. Error computations are based on the testing sample.

Table (2) is a summary of the applied Neural Networks model.

The table shows that the ratio of wrong rating ratio in the training sample reaches $9 \%$, while the wrong rating ratio in the test sample reaches $6.7 \%$, which are both nearly identical percentages. This indicates that the Network has been well trained on rating new terms. The table also shows that the applied Stopping Rule of the Network training is when the wrong rating ratio becomes constant or when the wrong ratio stops increasing, which depends on the test sample, as shown by the previous table that the Network training time is 25 seconds.

\begin{tabular}{|ll|c|c|c|}
\hline \multirow{3}{*}{ Sample } & Observed & Predicted \\
\cline { 3 - 5 } & & Admitted & Declined & $\begin{array}{l}\text { Correct } \\
\text { Percent }\end{array}$ \\
\hline \multirow{3}{*}{ Training } & Admitted & 90 & 3 & $96.8 \%$ \\
& Declined & 10 & 41 & $80.4 \%$ \\
& Overall Percent & $69.4 \%$ & $30.6 \%$ & $91.0 \%$ \\
& Admitted & 37 & 2 & $94.9 \%$ \\
Testing & Declined & 2 & 19 & $90.5 \%$ \\
& Overall Percent & $65.0 \%$ & $35.0 \%$ & $93.3 \%$ \\
\hline
\end{tabular}

Dependent Variable: Admission

Table (3) shows the results of rating using the suggested Neural Networks Table (3) shows that the correct rating of the accepted is $96.8 \%$ in the training sample while it is $90.5 \%$ in the testing sample. The correct rating ratio of the non-accepted declined - is $80.4 \%$ in the training sample, while it is $90.5 \%$ in the testing sample. 


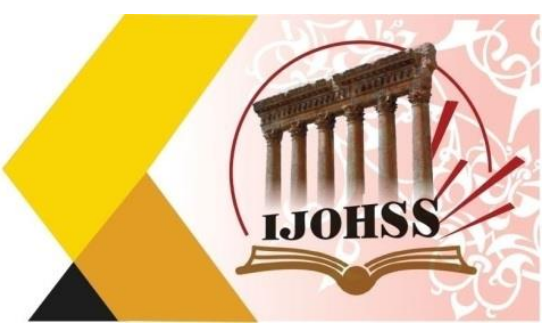

The testing sample shows that the correct rating ratio of the terms using this ANN reaches $93.3 \%$, which is considered an excellent prediction ratio by belonging or rating the new terms. The aforementioned shows also that the high ratio indicates the possibility of using the ANN for the purposes of rating with high efficiency.

Independent Variable Importance

\begin{tabular}{|l|l|l|}
\hline & Importance & $\begin{array}{l}\text { Normalized } \\
\text { Importance }\end{array}$ \\
\hline Places & .043 & $6.7 \%$ \\
Sex & .031 & $4.8 \%$ \\
Age & .061 & $9.4 \%$ \\
Scores in Arabic Language & .052 & $8.1 \%$ \\
Scores in Mathematics & .025 & $3.8 \%$ \\
Skills in Arabic Language & .045 & $7.0 \%$ \\
Material Expression & .025 & $3.8 \%$ \\
Verbal Abilities & .022 & $3.4 \%$ \\
Athletic Abilities & .014 & $2.1 \%$ \\
Wexler Test & .646 & $100.0 \%$ \\
Features Test & .036 & $5.6 \%$ \\
\hline
\end{tabular}

Table (4) the analysis of relative importance of independent variables

The above table shows the analysis of relative importance of independent variables whereas it shows that the most important variable influencing the rating with the use of the Neural Networks is Wexler's Test where as its importance reaches $64.6 \%$ followed by the age variable, $6.1 \%$, which is a very small percent as noted.

\section{Results:}

1. Rating the Neural Networks data has been $93.3 \%$, which is a high percent.

2. The technology of Neural Networks is characterized by arranging the order of independent variables according to their importance in influencing the dependent variable. This method is also characterized by not imposing restrictions on the dependent variable.

3. The most important factor influencing admission at the gifted schools is Wexler's Test.

\section{Recommendations:}

1. To use Neural Networks for the purpose of analysis

2. To use Neural Networks for the type of rating data.

3. To increase the accuracy of Neural Networks, the study recommends the introduction of Genetic Algorithm. 


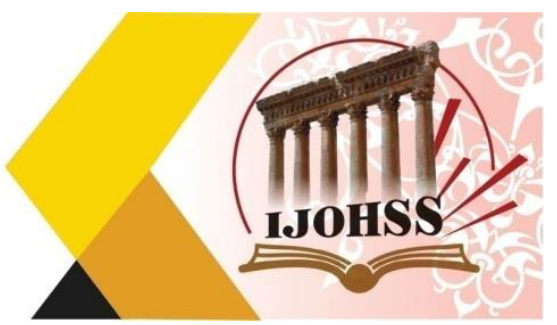

\section{References}

1. Everson , H. 1994, Using Artificial Neural Networks In Educational Research : some comparisons with statistical Models. Paper Presented At The Annul Meeting At The National Council on Measurement In Education. New Orleans, LA.

2. Gonzalez, j.M.B.\& Desjandins, S.S.L. 2001 Artificial Neural Networks; A new approach for predicting Appliaation Bchavior. Paper presented At The Annual Meeting of The Assoiration For Institutional Research.

3. http://unstats.un.org/unsd/demographic/products/socind/ststistics.

4. http:data.worldbank.org/indicator/NY.GDP.PCAP.CD/countries.

5. Khalafalla Ahmed Mohamed Arabi (2015) Factors affect Economic Growth Empirical Evidence from Sudan Economy International Journal of Research in Social Sciences Volume 5 Issue 1

6. Principe, C. jose, (2000) Neural and Adaptiv System Fundamental Through Simulations, John Wily \& Sons, Inc.,New York.

7. Smith, Anderw. (2004). "Branch Prediction with Neural Netwoks: Hidden layers and Recurrent Connectons" ,Department of Computer Science, University of California, San Diego, USA. 